\title{
Ets-family Transcription Factor Inhibitor TK216
}

National Cancer Institute

\section{Source}

National Cancer Institute. Ets-family Transcription Factor Inhibitor TK216. NCI

Thesaurus. Code C125657.

A proprietary biologic that inhibits the transcriptional-promoting activity of E26 transformation-specific (Ets, E-twenty-six) family transcription factors, with potential antineoplastic activity. Although the exact mechanism(s) of action through which this agent exerts its effect has yet to be fully elucidated, upon administration, Ets-family transcription factor inhibitor TK216 inhibits transcriptional activation mediated by Ets family proteins, including the oncogenic Ewing sarcoma breakpoint region 1/Friend leukemia virus integration 1 (EWSR1/FLI1; EWS/FLI1) fusion protein. This agent may both inhibit the malignant downstream effects mediated by genomic rearrangements that result in the overexpression of Ets family transcription factors and decrease tumor cell growth and proliferation. A chromosomal translocation $\mathrm{t}(11 ; 22)(\mathrm{q} 24 ; \mathrm{q} 12)$ fuses the EWSR1 gene and the FLI1 gene and encodes the EWSR1/FLI1 fusion protein, which is an oncoprotein expressed by peripheral primitive neuroectodermal (pPNET) tumors. 